\title{
Efeitos sedativos da associação de Cetamina e Midazolam administrados pela via intranasal ou intramuscular em papagaio (Amazona aestiva e Amazona vinacea) ${ }^{1}$
}

\author{
Eduarda H. Bitencourt ${ }^{2}$, Vanessa S. Padilha², Marcos Paulo A. de Lima², Suzane L. Beier ${ }^{3}$, \\ Aury N. de Moraes ${ }^{2}$ e Nilson Oleskovicz ${ }^{2 *}$
}

\begin{abstract}
Bitencourt E.H., Padilha V.S., Lima M.P.A., Beier S.L., Moraes A.N. \& Oleskovicz N. 2013. [Sedative effects of the association Ketamine and Midazolam administered intranasally or intramuscular in parrots (Amazona aestiva and Amazona vinacea).] Efeitos sedativos da associação de Cetamina e Midazolam administrados pela via intranasal ou intramuscular em papagaio (Amazona aestiva e Amazona vinacea). Pesquisa Veterinária Brasileira 33(9):1125-1129. Hospital de Clínicas Veterinárias, Centro de Ciências Agroveterinárias, Universidade do Estado de Santa Catarina, Av. Luiz de Camões 2090, Lages, SC 88520-000 Brazil. E-mail: a2no@cav.udesc.br

The lack of safe sedation protocols for use in parrots in the literature, demonstrate the need to know that the anesthetics are effective in these animals. Due to low muscle mass this bird, it was noted the need to consider other routes of administration, less invasive and painful to the animal, such as intranasal. The aim of this study was to evaluate sedative effects of intranasal administration compared to intramuscular $15 \mathrm{mg} \mathrm{kg}^{-1}$ of Ketamine and Midazolam 1mg.kg-1. We used 14 parrots (Amazona aestiva and Amazona vinacea), adults, and mean weight of $388.5 \pm 29.1 \mathrm{~g}$. The animals were randomly into two groups: intramuscular (IM, n=7) and intranasal (IN, n=7). In group intramuscular, administration of anesthetics was performed in the pectoral muscles, using insulin syringes and intranasal group with a micropipette. We evaluated the latency period, duration, quality of sedation, and the total recovery time. The average for the period of latency in the IM group was $6.13 \pm 2.02$ and IN group $4.84 \pm 2.37$ minutes. As for the duration of sedation in the IM group was $35.81 \pm 29.56$ and in IN group $24.52 \pm 14.83$ minutes. Both pathways promoted adequate sedation, the mean score for the quality of sedation obtained by the IM group was $2 \pm 1.5$ and $1.28 \pm 1.1$ in the IN group. The total recovery time in the IM group was $27.04 \pm 11.69$ and $17.67 \pm 11.64$ minutes in the IN group. Although the IN group the lowest times of onset, duration and full recovery and have better scores on quality of sedation, there was no statistically significant difference between groups. The results of this study indicate that administration of ketamine $\left(15 \mathrm{mg} \cdot \mathrm{kg}^{-1}\right)$ and midazolam $\left(1 \mathrm{mg} \cdot \mathrm{kg}^{-1}\right)$ intranasal or intramuscular in parrots (Amazona aestiva/Amazona vinacea) produce adequate sedation for minor procedures, but the intranasal route could be an alternative less invasive when compared to intramuscular injection.
\end{abstract}

INDEX TERMS: Anaesthesia, parrot, ketamine, midazolam, intranasally, intramuscular.

\footnotetext{
${ }^{1}$ Recebido em 5 de outubro de 2012.

Aceito para publicação em 25 de junho de 2013.

${ }^{2}$ Departamento de Medicina Veterinária, Centro de Ciências Agroveterinárias (CAV), Universidade do Estado de Santa Catarina (UDESC), Av. Luís de Camões 2090, Lages, SC 88520 000, Brasil. *Autor para correspondência: a2no@cav.udesc.br

${ }^{3}$ Departamento de Clínica e Cirurgia Veterinária, Escola de Veterinária, Universidade Federal de Minas Gerais (UFMG), Av. Antônio Carlos 6627, Pampulha, Belo Horizonte, MG 31270 901, Brasil.
}

RESUMO.- A falta de protocolos de sedação seguros para uso em papagaios na literatura demonstra a necessidade de conhecer os anestésicos que são eficazes nestes animais. Devido a pouca massa muscular desta espécie, notou-se a necessidade de estudar outra via de administração, menos invasiva e dolorosa ao animal, como a via intranasal. O objetivo deste estudo foi avaliar os efeitos sedativos e a viabilidade da administração intranasal, em comparação à 
via intramuscular, de $15 \mathrm{mg} / \mathrm{kg}$ de Cetamina e $1 \mathrm{mg} / \mathrm{kg}$ de Midazolam. Foram utilizados 14 papagaios das espécies Amazona aestiva e Amazona vinacea, de ambos os sexos, adultos, peso médio de $388,5 \pm 29,1 \mathrm{~g}$. Os animais foram distribuídos aleatoriamente em dois grupos: intramuscular (IM, n=7) e intranasal (IN, n=7). No grupo intramuscular, a administração dos anestésicos foi realizada nos músculos peitorais, utilizando seringas de insulina e no grupo intranasal, com auxílio de uma micropipeta. Avaliou-se o período de latência, tempo de duração, qualidade de sedação, e o tempo de recuperação total. A média para o período de latência no grupo IM foi de 6,13 $\pm 2,02$ minutos e no grupo

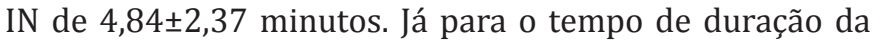
sedação no grupo IM a média foi de $35,81 \pm 29,56$ e no grupo IN de $24,52 \pm 14,83$ minutos. Ambas as vias promoveram sedação adequada, pois a média do escore da qualidade de sedação obtida pelo grupo IM foi $2 \pm 1,5$ e pelo grupo IN $1,28 \pm 1,1$. 0 tempo de recuperação total no grupo IM foi de $27,04 \pm 11,69$ e no grupo IN de $17,67 \pm 11,64$ minutos. Apesar do grupo IN ter apresentado os menores tempos de período de latência, duração e de recuperação total e ter obtido melhor escore na qualidade de sedação, não houve diferença estatística significativa entre os grupos. Os resultados obtidos neste estudo indicam que a administração de $15 \mathrm{mg} / \mathrm{kg}$ de cetamina e $1 \mathrm{mg} / \mathrm{kg}$ de midazolam pela via intranasal ou intramuscular em papagaios (Amazona aestiva e Amazona vinacea) produzem sedação adequada para pequenos procedimentos como colocação de anilha, coleta de sangue e radiografias; porém a via intranasal mostrou ser uma alternativa menos invasiva quando comparado à via intramuscular.

TERMOS DE INDEXAÇÃO: Anestesia, papagaios, cetamina, midazolam, intranasal, intramuscular.

\section{INTRODUÇÃO}

0 uso de agentes anestésicos em aves domésticas cresceu muito na última década (Uzun et al. 2003), principalmente devido ao fato de estarem se tornando animais de companhia, e como resultado dessa tendência, é comum o anestesista veterinário ser confrontado com pacientes que requerem um procedimento anestésico seguro e efetivo para uma ampla variedade de situações clínicas (Hall et al. 2001). Além dos que vivem como animais de estimação, muitos psitacídeos estão em extinção. Estes fatos reafirmam a necessidade de mais estudos nestas espécies, com o intuito de diminuir os riscos quando houver necessidade de algum procedimento anestésico (Paula, 2006). É importante avaliar as possibilidades anestésicas, particularmente para a anestesia geral com agentes injetáveis, já que em algumas situações não há possibilidade de empregar anestesia inalatória (Muresan et al. 2008).

A contenção manual é necessária para permitir procedimentos terapêuticos e diagnósticos, incluindo avaliação física, colheita de sangue e exames radiográficos (Mans et al. 2012). Um bom protocolo de sedação é essencial para diminuir o estresse e caso isso não ocorra, os animais podem vir a óbito em decorrência de complicações, especialmente quando doentes ou debilitados (Hawkins \& Pascoe 2007).
Além disto, a manipulação, sem prévia sedação, pode estimular respostas fisiológicas à situação estressante, levando a alterações que devem ser monitoradas, como aumento da frequência respiratória e temperatura (Greenacre \& Lusby 2004). Portanto, a contenção química permite que o médico veterinário realize uma avaliação segura, podendo proporcionar uma intervenção terapêutica apropriada (Al-Sobayil et al. 2009)

Os anestésicos injetáveis são frequentemente utilizados na anestesia de aves, dentre algumas de suas vantagens pode-se citar: o baixo custo, facilidade de administração, rápida indução anestésica, não requerer equipamentos específicos para administração e manutenção da anestesia, além de não poluírem o meio ambiente (Ludders \& Matthews 2007). No entanto, face ao reduzido tamanho dos animais e calibre dos vasos, a injeção intravenosa torna-se difícil ou até mesmo impraticável, sendo as vias intramuscular e subcutânea empregadas para a administração dos fármacos.

0 músculo peitoral é rotineiramente utilizado para injeções intramusculares, porém apresenta o grande risco de injeção intravascular acidental ou intracelomática em virtude do pequeno tamanho das aves (Kamiloglu et al. 2007). Além do mais, a administração intramuscular requer altas doses e pode apresentar absorção variável com o local da aplicação (Valverde et al. 1993). Neste sentido, Vesal \& Eskandari (2006) relataram que a via intranasal é um método não invasivo e viável como alternativa à administração intramuscular e subcutânea de fármacos em periquitos. As principais desvantagens do uso de anestésicos injetáveis em aves são as variações que ocorrem entre as espécies em relação aos efeitos farmacológicos, tornando-se difícil o ajuste seguro de doses, a outra dificuldade é a manutenção da anestesia cirúrgica que leva a depressão cardiorrespiratória, sendo frequentes as recuperações anestésicas prolongadas e violentas (Mostachio et al. 2008).

A cetamina é amplamente utilizada para uso em todas as espécies primatas, em muitos animais exóticos, como aves e répteis (Green et al. 1981), principalmente porque pode ser administrada por via intramuscular ou por via intravenosa, o que torna prática sua utilização em animais de difícil acesso venoso (Ajadi et al. 2009). Raramente a cetamina é utilizada de forma isolada, por causar pobre relaxamento muscular, tremores, mioclonias, opistótono e recuperação tempestuosa, por isso, é comumente associada com sedativos como os alfa ${ }_{2}$-agonistas, benzodiazepínicos ou azaperone, dependendo da espécie envolvida (Valverde et al. 1990).

Com base no exposto, este estudo tem como objetivo avaliar os efeitos sedativos, bem como a viabilidade da associação de Cetamina e Midazolam pelas vias Intramuscular (IM) e Intranasal (IN) em papagaios das espécies Amazona aestiva e Amazona vinacea.

\section{MATERIAL E MÉTODOS}

Este estudo foi aprovado pelo Comitê de Ética e Bem Estar Animal (CETEA) do Centro de Ciências Agroveterinárias (CAV) da Universidade do Estado de Santa Catarina (UDESC), protocolo 1.21.11.

Foram utilizados 14 papagaios das espécies Amazona aestiva 


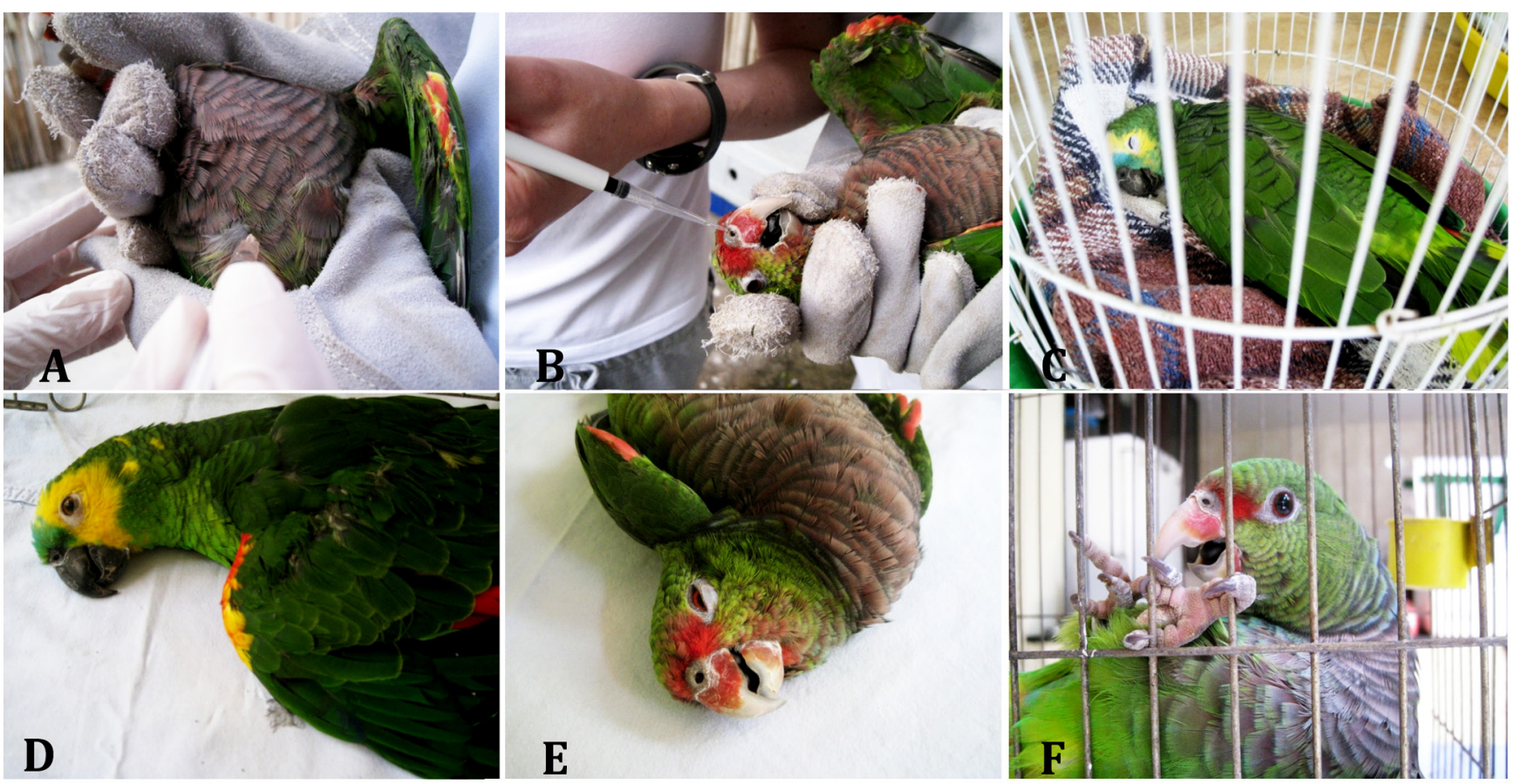

Fig.1. (A) Administração intramuscular de cetamina e midazolam. (B) Administração intranasal de cetamina e midazolam. (C) Escore de sedação 0, após administração de cetamina e midazolam. (D) Escore de sedação 1, após administração de cetamina e midazolam. (E) Escore de sedação 2, após administração de cetamina e midazolam. (F) Recuperação total após administração de cetamina e midazolam em papagaio (Amazona aestiva/Amazona vinacea).

e Amazona vinacea, de ambos os sexos, adultos, com peso médio de $388,5 \pm 29,1 \mathrm{~g}$ no grupo intramuscular e $382,8 \pm 49,5 \mathrm{~g}$ no grupo intranasal, oriundos de um Criatório Comercial Particular localizado na Rua da Hortências, 444, Jardim Janaína, Biguaçu, licença do Ibama protocolo no 02026.000417/2006-11.

As aves foram capturadas, sempre pela mesma pessoa, com auxílio de puçá no ambiente rotineiro, pesados e contidos manualmente com o uso de luvas de couro e posteriormente colocados em gaiolas individuais. Os papagaios foram alocados aleatoriamente em dois grupos $(n=7)$, sendo que os animais do grupo Intranasal (IN) receberam como protocolo sedativo $1 \mathrm{mg} / \mathrm{kg}$ de midazolam (Dormire ${ }^{\circledR}, 5 \mathrm{mg} / \mathrm{mL}$, Cristália Produtos Químicos e Farmacêuticos, Itapira/SP, Brasil), e $15 \mathrm{mg} / \mathrm{kg}$ de Cetamina (Vetaset $^{\circledR}, 100 \mathrm{mg} / \mathrm{ml}$, Fort Dodge Saúde Animal, São Paulo, Brasil), e do grupo Intramuscular (IM) a mesma associação pela via intramuscular. As doses foram estabelecidas através de estudos pilotos prévios ao início do estudo.

No IM, a administração dos anestésicos foi realizada nos músculos peitorais, utilizando seringas e agulhas de insulina (Fig.1 A), no IN a administração foi realizada com auxílio de uma micropipeta (Fig.1B) (Discovery Comfort HT volume de 20-200 $\mu \mathrm{L}$ ), administrando-se lentamente o volume calculado para cada animal, após contenção manual em decúbito dorsal, sendo primeiramente administrado a cetamina na narina direita, seguida imediatamente da administração de midazolam na narina esquerda.

Após a administração dos fármacos os animais foram colocados em decúbito esternal (Fig.1C) e em gaiolas individuais, dando-se início ao período de avaliação dos parâmetros. 0 período de latência foi definido pelo período de tempo entre o momento da aplicação dos fármacos até a presença do efeito clínico caracterizado pelo decúbito. 0 tempo de sedação, período de tempo entre o início da sedação caracterizada por redução dos reflexos protetores, olhos fechados, permanência em decúbito dorsal até o retorno dos primeiros reflexos. Em relação à qualidade da sedação, o animal foi observado continuamente por dois avaliadores cegos aos tratamentos, desde a aplicação dos fármacos até 15 minutos após a administração dos mesmos, atribuindo-se neste momento um único escore de sedação para cada animal, conforme escala de sedação própria, padronizada pelos autores: $(0)$ considerado a melhor sedação, com os olhos fechados, em decúbito, relaxado e sem movimentação; (1) olho aberto, em decúbito, relaxado, porém com pouca movimentação; (2) olho aberto piscando, em decúbito e relaxado; (3) olho aberto piscando, em pé. 0 tempo de recuperação total foi definido pelo tempo necessário para a presença de movimentos de cabeça, permanência do animal em poleiro sem sinais de desequilíbrio, movimentos coordenados e procura por comida.

Os dados quantitativos para comparação entre grupos foram submetidos ao teste $t$, para a qualidade de sedação foi utilizado o teste Mann Whitney Rank Sum Test $(\mathrm{P} \leq 0,05)$.

\section{RESULTADOS E DISCUSSÃO}

O período de latência no grupo IM foi de 6,13 $\pm 2,02$ minu-

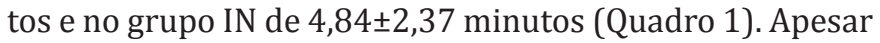
do grupo IN apresentar um menor período de latência, não houve diferença estatística significativa entre os grupos. Por não demonstrar diferenças estatísticas, a via intranasal demonstrou ser um método não invasivo aceitável para administração de fármacos. A via intranasal (transmucosa) é uma via alternativa tão eficaz quanto as vias intravenosa e intramuscular, devido a sua rica vascularização e alta permeabilidade, permitindo rápida absorção, sem que ocorra o efeito de primeira passagem pelo fígado (Kushwaha et al. 2011) Segundo Vesal \& Zare (2006), este método não requer nenhuma técnica especial e apenas necessita de um curto período de contenção para a administração dos fármacos, não havendo complicações ou reações adversas (Moghadam et al. 2009). Segundo Mulcahy (2007), o rápido 
Quadro 1. Valores médios e desvios-padrão do tempo de latência, tempo total de sedação, tempototal de recuperação e número de animais com os escores de sedação, após a administração de cetamina $15 \mathrm{mg} / \mathrm{kg}$ ) e midazolam (1 mg/ $\mathrm{kg}$ ) pela via intramuscular (IM) ou intranasal (IN) em papagaios (Amazona aestiva e Amazona vinacea)

\begin{tabular}{lcc}
\hline \multicolumn{1}{c}{ Variável } & Grupo & Resultado \\
\hline Tempo de latência (minutos) & IM & $6,13 \pm 2,02$ \\
Tempo total de sedação (minutos) & IN & $4,84 \pm 2,37$ \\
Tempo de recuperação total (minutos) & IM & $35,81 \pm 29,56$ \\
& IN & $24,52 \pm 14,83$ \\
Escore 0 de sedação & IM & $27,04 \pm 11,69$ \\
& IN & $17,67 \pm 11,64$ \\
Escore 1 de sedação & IM & $14,3 \%(1 / 7)$ \\
& IN & $28,6 \%(2 / 7)$ \\
Escore 2 de sedação & IM & $28,6 \%(2 / 7)$ \\
& IN & $28,6 \%(2 / 7)$ \\
Escore 3 de sedação & IM & $28,6 \%(2 / 7)$ \\
& IN & $28,6 \%(2 / 7)$ \\
& IM & $28,6 \%(2 / 7)$ \\
& IN & $14,3 \%(1 / 7)$
\end{tabular}

Escore de sedação: (0) considerado a melhor sedação, com os olhos fechados, em decúbito, relaxado e sem movimentação; (1) olho aberto, em decúbito, relaxado porém com pouca movimentação; (2) olho aberto piscando, em decúbito e relaxado; (3) olho aberto piscando, em pé.

início e tempo de ação dos fármacos administrados pela via intranasal em aves tornam essa via eficaz para ser utilizada na redução do estresse durante a captura de aves selvagens para à anestesia e cirurgia.

0 grupo IM apresentou tempo de duração da sedação de 35,81 29,56 minutos, com escore de sedação em média de 2 (olho aberto piscando, em decúbito e relaxado) , sendo que apenas um dos animais obteve escore 0 e dois animais não sedaram (Quadro 1). 0 grupo IN apresentou menor

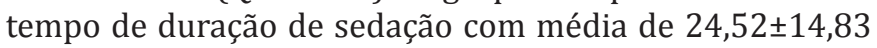
minutos e melhor qualidade de sedação, pois foi obtido sedação em todos os animais, sendo que dois apresentaram escore de sedação 0 (Fig.1C); dois apresentaram escore de sedação 1 (Fig.1D); dois com escore 2 (Fig.1E) e apenas um escore 3. No entanto, não foram observadas diferenças estatísticas significativas entre os grupos IM e IN para qualidade e duração da sedação.

Os resultados do tempo de sedação obtidos justificam-se pela associação dos fármacos utilizada, sendo a cetamina o agente de escolha para contenção química de diversas espécies de animais. Entretanto, é raramente utilizada de forma isolada em aves, principalmente pela ocorrência de mioclonias e pobre relaxamento muscular, podendo ser controladas com a associação de benzodiazepínicos ou agonistas alfa-2 adrenérgicos (Valverde et al. 1993). Da mesma forma, Green et al. (1981) afirmam que, em galinhas domésticas (Gallus gallus domesticus) a administração conjunta de cetamina $(25 \mathrm{mg} / \mathrm{kg})$ e diazepam $(2-2,5 \mathrm{mg} / \mathrm{kg})$ apresentou adequada sedação, levando alguns animais à anestesia cirúrgica com curto período de latência. De acordo com Soares (2007), após cinco minutos da administração intramuscular de $30 \mathrm{mg} / \mathrm{kg}$ de Cetamina e $2 \mathrm{mg} / \mathrm{kg}$ de midazolam, em galos (Gallus gallus domesticus), foi possível realizar a intubação endotraqueal.

Os resultados de Varner et al. (2004) demostraram que a combinação de cetamina e diazepam (20mg/kg e $2 \mathrm{mg} /$ $\mathrm{kg}$, respectivamente) aplicado por via intramuscular foi ineficiente para induzir à anestesia cirúrgica em galinhas. Por outro lado, de acordo com Paula (2006), a cetamina, na dose de $10 \mathrm{mg} / \mathrm{kg}$, associada ao diazepam, na dose de $0,5 \mathrm{mg} / \mathrm{kg}$, pela via intramuscular promoveu uma boa qualidade de sedação quando comparada ao agente inalatório sevofluorano; mostrando-se segura, não alterando as funções cardiovasculares, oxigenação ou ventilação, podendo ser utilizado com sucesso em papagaios. Segundo Vesal \& Eskandari (2006), o midazolam $(3,65 \mathrm{mg} / \mathrm{kg})$ administrado pela via intranasal, isolado ou em combinação com cetamina (40-50mg/kg), apresentou um período de latência de 2,7 minutos e produziu sedação que permitiu decúbito dorsal e sedação adequada para pequenos procedimentos em periquitos (Psittacus krameri).

Como demonstrado no Quadro 1, alguns animais não apresentaram sedação adequada, fato este que pode ser decorrente do ambiente em que foi realizada a aplicação dos fármacos, que não isolava os ruídos dos outros animais do recinto. 0 mesmo foi descrito por Lumeiji \& Deenik (2003) que ao compararem a associação de cetamina e medetomidina ou cetamina e diazepam em pombos, descreveram que os resultados de sedação podem sofrer interferência pela presença de estímulos externos (ruídos e atividade de outros animais), inexperiência do administrador, ou de administração inapropriada. Além disso, no grupo intranasal, o animal que obteve escore de sedação 3 espirrou logo após a administração dos fármacos, desta forma, podendo interferir na absorção do fármaco.

O tempo de recuperação total no grupo IM foi de $27,04 \pm 11,64$ minutos e no grupo IN foi de $17,67 \pm 11,69 \mathrm{mi}-$ nutos. Resultados semelhantes foram demonstrados por Azizpour \& Hassani (2012), em que a administração intramuscular de $10 \mathrm{mg} / \mathrm{kg}$ de cetamina e $0,2 \mathrm{mg} / \mathrm{kg}$ de diazepam em pombos promoveu lenta e suave recuperação, em $23,12 \pm 2,85$ minutos.

\section{CONCLUSÃO}

A administração de $15 \mathrm{mg} / \mathrm{kg}$ de cetamina e $1 \mathrm{mg} / \mathrm{kg}$ de midazolam pela via intranasal ou intramuscular em papagaios (Amazona aestiva e Amazona vinacea) promove sedação adequada (escore 1 e 2 respectivamente) para realização de pequenos procedimentos como colocação de anilha, coleta de sangue e radiografias, além do mais a via intranasal, mostrou ser uma alternativa menos invasiva quando comparado à via intramuscular.

\section{REFERÊNCIAS}

Al-Sobayil F.A., Ahmed A.F., Al-Wabel N.A., Al-Thinayian A.A., Al-Rogibah F.A., Al-Fuaim A.H., Al-Obaid A.O. \& Al-Muzaini A.M. 2009. The use of xylazine, ketamine, and isoflurane for induction and maintenance of anesthesia in Ostriches (Struthio camelus). J. Avian Med. Surg. 23(2):101-107.

Ajadi R.A., Kasali O.B., Makinde A.F., Adeleye A.I., Oyewusi J.A. \& Akintunde O.G. 2009. Effects of Midazolam on Ketamine-Xylazine Anesthesia in Guinea Fowl (Numida meleagris galeata). J. Avian Med. Surg. 23(3):199-204.

Azizpour A. \& Hassani Y. 2012. Clinical evaluation of general anaesthesia in pigeons using a combination of ketamine and diazepam. J. S. Afr. Vet. Assoc. 83(1):1-4. 
Green C.J., Knight J., Precious S. \& Simpkin S. 1981. Ketamine alone and combined with diazepam or xylazine in laboratory animals: a 10 year experience. Laboratory Animals 15:163-17.

Greenacre C.B. \& Lusby A.L. 2004 Physiologic responses of Amazon parrots (Amazona species) to manual restraint. J. Avian Med. Surg. 18(1):1922.

Hall L.W., Clarke K.W. \& Trim C.M. 2001. Veterinary Anaesthesia. $10^{\text {th }}$ ed. W.B. Saunders, London, p.472-474.

Hawkins M.G. \& Pascoe P.J. 2007. Cagebirds, p.281. In: West G., Heard D. \& Caulkett N. (Eds), Zoo Animal and Wildlife Immobilization and Anesthesia. Blackwell Publishing, Oxford, USA. 718p.

Kamiloglu A., Atalan G. \& Kamiloglu N.N. 2007. Comparison of intraosseous and intramuscular drug administration for induction of anaesthesia in domestic pigeons. Res. Vet. Sci. 85:171-175.

Kushwaha S.K.S., Keshari R.K. \& Rai A.K. 2011. Advances in nasal trans-mucosal drug delivery. J. Appl. Pharmaceut. Sci. 1(7):21-28.

Ludders J.W. \& Matthews N.S. 2007. Birds, p.841-868. In: Tranquilli W.J., Thurmon J.C. \& Grimm K.A. (Eds), Lumb and Jones, Veterinary Anesthesia and Analgesia, $4^{\text {th }}$ ed. Blackwell Publishing, Oxford, USA. 1096p.

Lumeij J.T. \& Deenik J.W. 2003. Medetomidine-ketamine and diazepamketamine anesthesia in racing pigeons (Columba livia domestica): a comparative study. J. Avian Med. Surg. 17(4):191-196.

Mans C., Guzman D.S., Lahner L.L., Paul-Murphy J. \& Sladky K.K. 2012. Sedation and physiologic response to manual restraint after intranasal administration of midazolam in hispaniolan Amazon parrots (Amazona ventralis). J. Avian Med. Surg. 26(3):130-139.

Moghadam A.Z., Sadegh A.B., Sharifi S. \& Habibian S. 2009. Comparison of intranasal administration of diazepam, midazolam and xylazine in Pigeons: Clinical evaluation. Iranian J. Vet. Sci. Technol. 1(1):16-26.

Mostachio G.Q., Oliveira L.D., Carciofi A.C. \& Vicente W.R.R. 2008. The effects of anesthesia with a combination of intramuscular xylazine-diazepam-ketamine on heart rate, respiratory rate and cloacal temperature in roosters. Vet. Anaesth. Analg. 35:232-236.
Mulcahy D.M. 2007. Free-living waterfow land shorebirds, p.318. In: West G., Heard D. \& Caulkett N. (Eds), Zoo Animal and Wildlife Immobilization and Anesthesia. Blackwell Publishing, Oxford, USA. 718p.

Muresan C., Czirjak G.A., Pap P.L. \& Kobolkuti L.B. 2008. Ketamine and Xylazine anaesthesia in the house sparrow. Vet. Med. 65(2):193-195.

Paula V.V. 2006. Efeito da pré-medicação em papagaios (Amazona aestiva), com cloridrato de cetamina isolado ou associado ao diazepam, sobre a indução e anestesia com sevofluorano. Tese em Clínica Cirúrgica Veterinária, Faculdade de Medicina Veterinária e Zootecnia, Universidade de São Paulo, SP. 126p.

Soares A.V., Pippi N.L. \& Freitas G.C. 2007. Cetamina e midazolam como medicação anestésica indutora e manutenção com isofluorano e oxigênio a $100 \%$ para cirurgia em galo (Gallus gallus domesticus): relato de caso. Vet. Notícias 13(1):81-84.

Uzun M., Yildiz S., Atalan G., Kaya M. \& Sulu N. 2003. Effects of Medetomidine-Ketamine combination anaesthesia on electrocardiographic findings, body temperature, and heart and respiratory rates in domestic pigeons. Turkish J. Vet. Anim. Sci. 27:377-382.

Valverde A., Bienzle D., Smith D.A., Dyson D.H. \& Valliant A. 1993. Intra-osseous cannulation and drug administration for induction of anaesthesia in chicken. Vet. Surg. J. 22(3):240-244.

Valverde A., Honeyman V.L., Dyson D.H. \& Valliant A.E. 1990. Determination of a sedative dose and influence of midazolam on cardiopulmonary function in Canada geese. J. Am. Vet. Med. Assoc. 51(7):1071-1074.

Varner J., Clifton K.R., Poulos S., Broderson J.R. \& Wyatt R.D. 2004. Lack of efficacy of injectable Ketamine with Xylazine or Diazepam for anesthesia in chickens. Lab. Animals 33(5):36-39.

Vesal N. \& Eskandari M.H. 2006. Sedative effects of midazolam and xylazine with or without ketamine and detomidine alone following intranasal administration in Ring-necked Parakeets. J. Am. Vet. Med. Assoc. 228:383-388.

Vesal N. \& Zare P. 2006. Clinical evaluation of intranasal benzodiazepines, $\alpha_{2}$-agonists and their antagonists in canaries. Vet. Anaesth. Analg. 33:143-148. 